\title{
Autoeficacia, Apoyo Social y Calidad de Vida en Adolescentes con Enfermedades Crónicas ${ }^{1}$
}

\author{
Self-Efficacy, Social Support and Quality of Life of Adolescents with Chronic Illnesses
}

\author{
María José Avendaño Monje \\ Enrique Barra Almagiá \\ Universidad de Concepción, Chile \\ (Rec: 15 de enero 2008 Acep: 06 de noviembre 2008)
}

\begin{abstract}
Resumen
Este estudio tuvo como objetivo examinar la relación existente entre la calidad de vida reportada por pacientes adolescentes con enfermedades crónicas y dos variables consideradas relevantes para el bienestar y la salud, la autoeficacia y el apoyo social percibido. Los participantes fueron 75 adolescentes de ambos sexos, entre 12 y 18 años, con diagnóstico de diabetes, fibrosis quística e insuficiencia renal, pacientes del Hospital Regional de Concepción. Los instrumentos utilizados fueron el Cuestionario de Calidad de Vida KIDSCREEN-27, la Escala de Autoeficacia Generalizada y el Cuestionario de Apoyo Social Percibido. Se encontró que la autoeficacia tenía una influencia muy significativa en el nivel de calidad de vida, explicando un tercio de la varianza, mientras que el apoyo social percibido mostró tener muy poca influencia sobre la calidad de vida informada por los adolescentes. En cuanto a la variable género, la única diferencia significativa fue que los hombres informaron un mayor nivel de bienestar físico que las mujeres. Se discuten los resultados obtenidos a la luz de diversos factores explicativos.

Palabras clave: Adolescentes, enfermedad crónica, autoeficacia, apoyo social.
\end{abstract}

\begin{abstract}
This study was designed to estimate the relationships of self-efficacy and perceived social support to quality of life in adolescents with chronic illnesses. Participants were 75 male and female adolescents with diabetes, cystic fibrosis or renal failure, 12 to 18 years old, from Concepción (Chile). Individuals completed the Quality of Life measure KIDSCREEN-27, the General Self-Efficacy Scale and the Perceived Social Support Questionnaire. Results showed that quality of life reported by adolescents had a very significant positive relationship with self-efficacy and a lower relationship with social support. The only significant gender difference in the variables examined was that adolescent females showed a lower level of physical well-being than males. Diverse explanatory factors for obtained results are proposed. .
\end{abstract}

Key words: Adolescents, chronic illness, self-efficacy, social support.

1 Este artículo se basa en la Tesis de la primera autora para optar al grado de Magíster en Psicología de la Salud de la Universidad de Concepción. Correspondencia: María José Avendaño Monje, Departamento de Psicología, Universidad de Concepción, Casilla 160-C, Concepción, Chile. E-mail: maravendanom@udec.cl 


\section{Introducción}

El concepto de calidad de vida ha cobrado cada vez mayor relevancia en la Psicología de la Salud, debido entre otros factores a la transición demográfico-epidemiológica que enfrentan países como el nuestro y que implica un mayor tiempo de exposición de las personas a los factores de riesgo responsables de las patologías crónicas y una mayor expectativa de vida de quienes padecen tales enfermedades.

La calidad de vida puede definirse como la percepción subjetiva de bienestar general que resulta de la evaluación que hace el individuo de diversos dominios o áreas de su vida (Bishop, 2005). Se trata de un constructo multidimensional, que incorpora aspectos tales como la salud física de la persona, su estado psicológico, grado de independencia, relaciones sociales, factores ambientales y creencias personales.

Una enfermedad crónica es una condición de salud de duración extendida en el tiempo, mayor de 6 meses y a menudo de por vida, que implica algún grado de limitación en la vida cotidiana del individuo y puede conllevar discapacidad, con largos períodos de cuidado y supervisión, ya sea como consecuencia de su severidad y/o de los efectos del tratamiento (Yeo \& Sawyer, 2005).

La enfermedad crónica puede ser descrita como un evento estresante y un gran desafío adaptativo, que implica cambios en el estilo de vida y un proceso de ajuste, a veces de por vida, a diversos cambios físicos, psicológicos, sociales y ambientales que conlleva la enfermedad y su tratamiento, pudiendo eventualmente generar una incapacidad permanente en quien la padece (Bishop, 2005; Han, Lee, Lee \& Park, 2003).

Independiente de los recursos que posea el individuo, el diagnóstico de una enfermedad crónica resulta una experiencia inesperada, traumática y desestabilizadora (Grau, 2005). Por ello, es necesario desarrollar un proceso de adaptación a la enfermedad crónica, el que tendrá como uno de sus resultados, una determinada calidad de vida, siendo factores importantes para este resultado el curso del tratamiento, la limitación funcional, el estrés psicosocial asociado con la enfermedad, las características personales y del entorno socio-familiar (Livneh \& Antonak, 2005).

Por ser la adolescencia un tiempo de rápido crecimiento y cambios fisiológicos, acompañados por importantes procesos de individuación y socialización, el manejo de una condición crónica durante este período constituye un importante desafío para el individuo, su familia y el equipo de salud (Suris, Michaud \& Viner, 2004). Ello se debe a que la enfermedad crónica impacta las distintas esferas del desarrollo del adolescente, con efectos significativos en los aspectos físicos, emocionales, sociales, educacionales $\mathrm{y}$ vocacionales, por lo cual se puede considerar que las personas jóvenes con condiciones crónicas encaran más dificultades en la resolución de las tareas de la adolescencia que sus pares saludables (Yeo \& Sawyer, 2005).

Es por ello que para minimizar los efectos biopsicosociales que puede tener la enfermedad crónica y favorecer el desarrollo evolutivo normal del adolescente, se deben identificar aquellas características personales que promoverían el ajuste a la enfermedad y una mejor calidad de vida. Dentro de esas características estarían el sentido de autoeficacia y la percepción de apoyo social de parte de las personas significativas, además de la posible influencia que podrían tener algunos factores sociodemográficos tales como sexo y edad.

La auto-eficacia forma parte del auto-concepto y se desarrolla en interacción con el ambiente social y el proceso de individuación, dado que éste permite la adquisición de conocimientos acerca de uno mismo y el mundo externo, el desarrollo de competencias y, por esta vía, la adquisición del sentido de eficacia personal (Pastorelli, Caprara, Barbaranelli, Rola, Rozsa \& Bandura, 2001).

Los principales recursos de información de eficacia para los niños son la familia, los pares y la escuela. Estos recursos van apareciendo uno tras otro en la vida de un individuo, contribuyendo al desarrollo de un adecuado o inadecuado sentido de auto-eficacia y permitiendo la evolución desde el control externo hasta la autorregulación personal (Pastorelli, Caprara, Barbaranelli, Rola, Rozsa \& Bandura, 2001).

Según como se desarrolle la auto-eficacia, se definirá una determinada vulnerabilidad o resistencia al estrés $y$, por tanto, la capacidad de adaptación a las demandas que implican las transiciones evolutivas (Bandura, Pastorelli, Barbaranelli \& Caprara, 1999), así como aquellas que impone la experiencia de vivir con una condición de enfermedad crónica.

La investigación respecto al tema evidencia que las creencias de auto-eficacia jugarían un rol importante en el ajuste a las condiciones de salud crónica y por lo tanto en la calidad de vida resultante (Han, Lee, Lee, \& Park, 2003; Rose, Fliege, Hildebrandt, Schirop \& Klapp, 2002). Se ha informado que, independientemente del tipo y severidad de la enfermedad, la dimensión de autoeficacia se relaciona con la sintomatología física y el funcionamiento psicosocial tanto en adultos (Arnold et al., 2005; Kohler, Fish \& Greene, 2002; Wu, Tang \& Kwok, 2004) como en adolescentes (Grey, Boland, Yu, Sullivan-Bolyai \& Tamborlane, 1998). Así por ejemplo, en el estudio de Grey y colaboradores (1998) con adolescentes diabéticos se encontró que la menor calidad de vida tenía una relación significativa con una menor autoeficacia, así como con la percepción de dificultad en el manejo de la enfermedad, la utilización de estrategias de afrontamiento de rebelión y la sintomatología depresiva.

Otra característica habitualmente asociada a la salud y a la calidad de vida es el apoyo social, cuyos efectos sobre el bienestar y la salud se han conceptualizado desde dos 
modelos diferentes. Desde un modelo de efectos principales, se sostiene que el apoyo social favorece el bienestar y la salud independientemente de los niveles de estrés del individuo; por otra parte, desde el modelo de amortiguación se sostiene que el apoyo social sirve como una fuente de protección de los efectos patogénicos de los eventos estresantes muy intensos o prolongados (Barra, 2004). Independientemente del modelo al que se adhiera, la literatura en torno al tema muestra evidencias concluyentes en adultos, respecto a la influencia del apoyo social en la salud y en la calidad de vida en general y específicamente en personas con enfermedades crónicas (Gielen, McDonnell, Wu, O'Campo \& Faden, 2001; Han et al., 2003; Uchino, 2006).

Respecto a los adolescentes, aunque la evidencia es menos numerosa y concluyente, también se ha informado la existencia de una relación general entre apoyo social y estado de salud (Gecková, Van Dijk, Stewart, Groothoff \& Postt, 2003) y entre apoyo social y calidad de vida (Malkowska, Mazur, \& Woynarowska, 2004). En el estudio de Malkowska y colaboradores (2004), realizado con una muestra representativa de niños y adolescentes polacos de entre 8 y 18 años, se encontró que el nivel de apoyo social percibido tenía una influencia significativa sobre la calidad de vida. Los niños con menor apoyo social reportaron más baja calidad de vida en comparación con los que percibían mayor apoyo, especialmente en la dimensión de relaciones con los padres y vida en el hogar, por lo que se concluye que el apoyo social que proveen los padres incrementa la calidad de vida en niños y adolescentes. Este resultado se podría explicar porque los miembros de la familia proveen un recurso único de apoyo tanto en preocupaciones cotidianas como en situaciones de emergencia, a diferencia de los pares que resultan de poca ayuda en éstas últimas (Frey \& Röthlisberger, 1996).

Sin embargo, la evidencia acerca de la influencia que tendría el apoyo social sobre el bienestar y salud de los adolescentes sería menos consistente que en el caso de los adultos. Así por ejemplo, en nuestro medio y utilizando la misma medida de apoyo social empleada en el presente estudio, Barra, Cerna, Kramm y Véliz (2006) encontraron que el apoyo social percibido no se relacionaba con los problemas de salud informados por adolescentes sanos, así como tampoco con las otras variables del estudio -eventos estresantes, estrés percibido y ánimo depresivo-, las cuales sí mostraban relación significativa con los problemas de salud. Y en aquellos estudios que han encontrado relación entre apoyo social y estado de salud en adolescentes, tal relación tiende a ser más bien baja (Barra, Cancino, Lagos, Leal \& San Martín, 2005; Gecková et al., 2003)

En cuanto a niños y adolescentes con enfermedades crónicas, existen algunas evidencias acerca de los valiosos efectos del apoyo social sobre el bienestar individual y la calidad de vida (Pendley, Kasmen, Miller, Donze, Swenson \& Reeves, 2002; Vázquez et al., 2003; Woodgate, 2006). En un estudio prospectivo de Skinner, John y Hampson (2000) en adolescentes con diabetes tipo I, en el cual se buscaba determinar si el apoyo de pares y la representación de la enfermedad mediaba la asociación entre apoyo familiar, auto-cuidado y bienestar, se encontró que el impacto percibido de la diabetes y el apoyo de la familia y amigos fueron predictivos del nivel de bienestar de los participantes.

Con respecto a la posible influencia de factores sociodemográficos sobre la calidad de vida en adolescentes con enfermedades crónicas, existe gran escasez de evidencias y sólo recientemente se ha empezado a examinar la influencia del sexo y la edad en la calidad de vida relacionada con la salud. En cuanto a lo primero, en un estudio destinado a examinar si existían diferencias en la calidad de vida de pacientes adolescentes hombres y mujeres con fibrosis quística (Arrington, Yi, Tsevat, Wilmott, Mrus \& Britto, 2006), se encontró que las mujeres reportaban un nivel significativamente inferior en 5 de los 12 dominios de calidad de vida evaluados. Además, controlando las variables edad y fuerza del volumen espiratorio, las mujeres también obtuvieron menores puntajes en salud global, salud mental y percepción de salud general. Por su parte, en un estudio con alrededor de 300 niños y adolescentes (8-16 años) de 5 países europeos que padecían asma, diabetes y artritis, Petersen, Schmidt y Bullinger (2006) encontraron que de las 6 dimensiones evaluadas de la calidad de vida relacionada con la salud, las mujeres obtuvieron puntajes inferiores a los hombres en 2 dimensiones: Física y Emoción.

En cambio el panorama es menos claro respecto a la influencia de la edad en la mayor o menor calidad de vida de los adolescentes con enfermedades crónicas. En el estudio mencionado de Petersen y colaboradores (2006) se encontró un efecto significativo de la edad en sólo una de las 6 dimensiones (Emoción), siendo los participantes menores los que presentaban mayor puntaje. Sin embargo es necesario tomar en cuenta que en este estudio la edad de los participantes era de 8 a 16 años, por lo cual los menores de la muestra eran niños más que adolescentes.

A partir de la revisión de la literatura especializada se puede concluir que, en comparación con los adultos, la evaluación de la calidad de vida en pacientes adolescentes es un área de investigación mucho más reciente (Petersen et al., 2006), por lo cual aun queda mucho por conocer acerca del tema de la calidad de vida en los adolescentes con enfermedades crónicas y los factores que pueden influir en ella. Siendo la calidad de vida un concepto de trascendental importancia en las personas de cualquier edad que presentan una enfermedad crónica, aparece como especialmente relevante en la adolescencia, debido a la gran cantidad y variedad de cambios y tareas vitales que implica el desarrollo en esta etapa.

Debido a las variadas repercusiones psicosociales de la enfermedad crónica en especial durante la adolescencia, surge la necesidad de contar con más elementos para la 
promoción del desarrollo y crecimiento óptimo de los pacientes adolescentes, considerando la etapa evolutiva de cambio y transición en la que se encuentran. Tomando en cuenta la escasez de estudios, especialmente nacionales, que aborden las relaciones entre calidad de vida relacionada con la salud y factores personales, el presente estudio tuvo los siguientes objetivos:

Examinar la relación entre la calidad de vida y las variables de autoeficacia, apoyo social percibido y edad en adolescentes con enfermedades crónicas

Determinar posibles diferencias de género en la calidad de vida de los adolescentes con enfermedades crónicas.

\section{Método}

\section{Participantes}

Los participantes en este estudio fueron 75 adolescentes (38 hombres y 37 mujeres), con edades entre 12 y 18 años (promedio 15.4 años) con diagnóstico de diabetes mellitus, fibrosis quística e insuficiencia renal, atendidos en el Hospital Regional de Concepción. En cuanto al tiempo de evolución de la enfermedad, ésta fluctuaba entre 1 y 17 años, con un promedio de 6.9 años.

\section{Instrumentos}

Autoeficacia: se aplicó la Escala de Autoeficacia Generalizada (Schwarzer \& Scholz, 2000). Consta de 10 ítems referidos a afrontar exitosamente distintas situaciones. Posee 3 alternativas para contestar, "Más bien cierto", "Apenas Cierto" e "Incorrecto", por lo cual los puntajes pueden variar entre 10 y 30 puntos. Ha sido utilizada en diversas culturas, en población adulta y adolescente desde los 12 años de edad. Respecto a la confiabilidad por consistencia interna, se han obtenido en muestras de 23 naciones coeficientes alfa de Cronbach que van desde 0.76 a 0.90 . Respecto a su validez, se han encontrado relaciones positivas con emociones favorables, optimismo disposicional y satisfacción con el trabajo, y relaciones negativas con ansiedad, depresión y síntomas físicos. En el presente estudio se obtuvo un coeficiente de confiabilidad alfa de Cronbach de 0.79 .

Apoyo social percibido: se utilizó el Cuestionario de Apoyo Social Percibido (Gracia, Herrero \& Musitu, 2002), el cual incluye 6 ítems agrupados en 3 dimensiones de apoyo (emocional, consejo y ayuda). Primero el individuo debe indicar las iniciales de las personas relevantes para él o ella y luego debe contestar los 6 ítems referidos a cuánto apoyo percibe en cada una de esas personas relevantes, con cinco opciones de respuesta por ítem, desde 1 (NuncaCasi Nunca) hasta 5 (Siempre-Casi Siempre). Respecto a sus características psicométricas, los autores informan un coeficiente de consistencia interna alfa de Cronbach igual a 0.99 y evidencias de validez discriminante en adultos
(Gracia et al., 2002). En el presente estudio se obtuvo un coeficiente alfa de Cronbach de 0.84 .

Calidad de vida: Se utilizó el Cuestionario de Calidad de Vida relacionada con la Salud para Niños y Jóvenes KIDSCREEN-27, instrumento genérico que permite evaluar calidad de vida en niños y adolescentes entre 8 y 18 años. Este instrumento es una versión reducida del original KIDSCREEN-52 (Ravens-Sieberer et al., 2005) y comprende 27 ítems que se agrupan en 5 dimensiones de la calidad de vida: Bienestar Físico; Bienestar Psicológico; Autonomía y Relación con Padres; Amigos y Apoyo Social; y Entorno Escolar. El rango de puntuación de todos los ítems es de 1 a 5. Para la versión original, el KIDSCREEN-52, se informa una confiabilidad por consistencia interna mediante alfa de Cronbach que varía entre 0.77 y 0.89 . Respecto a su validez de constructo, se ha encontrado una alta correlación con el estado de salud de niños y adolescentes. En el presente estudio, se obtuvo un coeficiente alfa de Cronbach de 0,90.

\section{Procedimiento}

Luego de obtener la aprobación del Comité de Investigación y Ética del Hospital, se realizó una entrevista con cada adolescente y su tutor donde se les explicaba de modo general el objetivo del estudio y se solicitaba su colaboración. A quienes accedían a participar y firmaban las fichas de asentimiento y consentimiento se les aplicaron individualmente los instrumentos en una sesión de aproximadamente 45 minutos en dependencias del Hospital.

\section{Resultados}

Para cumplir con el primer objetivo, examinar la relación entre calidad de vida y las variables de autoeficacia, apoyo social percibido y edad en adolescentes con enfermedades crónicas, en primer lugar se calcularon los coeficientes de correlación de Pearson entre las variables estudiadas.

Tabla 1. Correlaciones entre las diversas variables

\begin{tabular}{lccc}
\hline & Autoeficacia & Apoyo social & Edad \\
\hline Calidad de vida & $.558^{* *}$ & $.283^{*}$ & $-.236^{*}$ \\
Autoeficacia & - & $.379^{* *}$ & .008 \\
Apoyo social & & - & .110 \\
\hline$* \mathrm{p}<0.05 ; * \mathrm{p}<0.01$ & & &
\end{tabular}

Como se puede observar en la Tabla 1, tanto la autoeficacia como el apoyo social percibido muestran una relación positiva significativa con la calidad de vida informada, sin embargo la relación entre esta última y la autoeficacia es claramente mayor a la relación entre calidad y vida y apoyo social. También se observa que la calidad de vida presenta una moderada relación inversa con la edad, es decir, en este grupo de adolescentes a mayor edad tiende a disminuir ligeramente la calidad de vida informada. 
Tabla 2. Coeficientes de regresión

\begin{tabular}{ccccccc}
\hline & $\begin{array}{c}\text { Coefic. Estandar. } \\
\text { Beta }\end{array}$ & $t$ & Sig. & $\begin{array}{c}\text { Correlaciones } \\
\text { Orden cero }\end{array}$ & Parcial & Semi-parcial \\
\hline Autoeficacia &, 517 & 5,114 &, 000 &, 558 &, 519 &, 478 \\
Apoyo social &, 115 & 1,134 &, 261 &, 283 &, 133 &, 106 \\
Edad &,- 253 & $-2,688$ &, 009 &,- 236 &,- 304 &,- 251 \\
\hline
\end{tabular}

Variable dependiente: Calidad de vida

El hecho de que la calidad de vida estaría mucho más relacionada con la autoeficacia que con el apoyo social queda más patente aun en los resultados del análisis de regresión que se presentan la Tabla 2, donde se puede observar que la autoeficacia tiene un peso mucho mayor sobre la calidad de vida que el apoyo social percibido. A pesar que este último tiene una correlación simple significativa con la calidad de vida, dicha relación no es significativa en términos de correlación parcial ni de regresión. Se puede apreciar que la variable edad tiene un peso mucho mayor que el apoyo social percibido sobre la calidad de vida, siendo en este caso la relación de tipo inverso, es decir, a mayor edad menor calidad de vida informada. Pero es la autoeficacia la variable que muestra tener la mayor influencia sobre la calidad de vida tanto en el análisis de correlación como en el de regresión. Tal como se puede observar en la Tabla 3 , el modelo de regresión que incluye sólo la autoeficacia explicaría casi un tercio de la varianza en la calidad de vida informada $(30 \%)$, si se le agrega la variable edad se produce un aumento de alrededor de un 5\% en la varianza explicada, en cambio la inclusión del apoyo social percibido no aumenta la capacidad explicativa del modelo de regresión.

Respecto al segundo objetivo, el examen de las diferencias de género en calidad de vida y en sus dimensiones mediante la prueba $\mathbf{t}$ de diferencias de medias revela que los hombres adolescentes presentan en general puntajes promedio superiores a las mujeres tanto en la calidad de vida global como en sus dimensiones, con la excepción de

Tabla 3. Resumen del modelo de regresión

\begin{tabular}{ccccccc}
\hline Modelo & $R$ & $R$ cuadrado & $\begin{array}{c}R \text { cuadrado } \\
\text { corregida }\end{array}$ & $\begin{array}{c}\text { Cambio en } R \\
\text { cuadrado }\end{array}$ & Cambio en $F$ & $\begin{array}{c}\text { Sig. del } \\
\text { cambio en } F\end{array}$ \\
\hline 1 &, $558(\mathrm{a})$ &, 312 &, 302 &, 312 & 33,031 &, 000 \\
2 &, 608 (b) &, 369 &, 352 &, 058 & 6,604 &, 012 \\
3 &, 617 (c) &, 381 &, 354 &, 011 & 1,286 &, 261 \\
\hline
\end{tabular}

Variable dependiente: Calidad de vida

a Variables predictoras: Autoeficacia

b Variables predictoras: Autoeficacia, Edad

c Variables predictoras: Autoeficacia, Edad, Apoyo social

Tabla 4. Variable dependiente: Calidad de vida

\begin{tabular}{lrrrrr}
\hline & \multicolumn{2}{c}{$\begin{array}{c}\text { Hombres } \\
(\mathrm{n}=38)\end{array}$} & \multicolumn{2}{c}{$\begin{array}{c}\text { Mujeres } \\
(\mathrm{n}=37)\end{array}$} & $\mathrm{t}$ \\
\hline Calidad de vida & 105,37 & 15,38 & 99,92 & 14,09 & 1,60 \\
Bienestar Físico & 17,92 & 4,10 & 15,57 & 3,790 & $2,58^{*}$ \\
Bienestar Psicológico & 27,58 & 4,28 & 25,89 & 4,80 & 1,60 \\
Autonomía y Relación & 27,63 & 5,04 & 27,16 & 4,56 &, 42 \\
con Padres & 17,05 & 2,97 & 16,03 & 3,35 & 1,39 \\
Amigos y Apoyo Social & 15,18 & 2,79 & 15,27 & 2,70 &,- 13 \\
Entorno Escolar & 25,34 & 3,44 & 25,51 & 3,330 &,- 21 \\
Autoeficacia & 4,14 &, 54 & 4,36 &, 50 & $-1,80$ \\
Apoyo social & & & & &
\end{tabular}


la dimensión Entorno Escolar. Sin embargo, como se puede observar en la Tabla 4, sólo en la dimensión de Bienestar Físico la diferencia de género a favor de los hombres llega a ser significativa. También se observa en la Tabla 4 que hay una diferencia no significativa a favor de las mujeres en apoyo social y que no existe diferencia de género en autoeficacia.

\section{Discusión}

Los resultados obtenidos en el presente estudio con adolescentes portadores de una enfermedad crónica muestran que la calidad de vida presenta una relación positiva muy significativa con la autoeficacia y una asociación positiva mucho menor con el apoyo social percibido, siendo esta última relación sólo algo mayor a la asociación negativa entre calidad de vida y edad. De acuerdo al análisis de regresión realizado, el nivel de autoeficacia de estos adolescentes puede ser considerado como un predictor significativo del nivel de calidad de vida informada, en cambio el nivel de apoyo social percibido parece no tener una influencia importante en la calidad de vida reportada.

El hecho de que la autoeficacia muestre tener una influencia significativa en la calidad de vida de este grupo de adolescentes con enfermedades crónicas es consistente con evidencias en adultos que muestran que la autoeficacia sería un factor crucial para la percepción de estado de salud, la calidad de vida general y la calidad de vida relacionada con la salud en pacientes con enfermedades crónicas (Kohler, Fish \& Greene, 2002; Kuijer \& De Ridder, 2003; Wahl, Rustoen, Hanestad, Gjengedal \& Moum, 2005). Así por ejemplo, en un estudio con pacientes con enfermedad pulmonar obstructiva crónica, Kohler y otros encontraron que la autoeficacia mediaba la relación entre la función pulmonar y la calidad de vida. Por su parte, en un estudio con pacientes portadores de fribosis quística en el cual se utilizó el mismo instrumento de autoeficacia generalizada empleado en la presente investigación, Wahl et al (2005) encontraron que la autoeficacia tenía una influencia directa tanto en el impacto percibido de la enfermedad (estado de salud) como en la calidad de vida global.

La influencia que tiene la autoeficacia sobre la calidad de vida de los adolescentes con enfermedades crónicas, se puede explicar por el hecho que un mayor nivel de autoeficacia producirá una mayor vulnerabilidad o resistencia al estrés, una mayor capacidad de adaptación a las demandas que implican las transiciones evolutivas y también una mayor capacidad de ajuste a la experiencia de vivir con una condición de enfermedad crónica. Esta explicación estaría apoyada por diversos estudios que plantean que la auto-eficacia favorece la calidad de vida, el bienestar emocional y el afrontar la adversidad en un amplio rango de situaciones estresoras y demandantes, como es el caso de vivir con una enfermedad crónica (Bandura et al., 1999;
Caprara, Steca, Gerbino, Paciello \& Vecchio, 2006; Grey et al., 1998; Luszczynska, Gutiérrez. \& Schwarzer, 2005).

El hecho de que en este grupo de adolescentes con enfermedades crónicas el apoyo social muestre baja relación con la calidad de vida se puede explicar porque en la adolescencia las estructuras de apoyo social experimentan importantes cambios, desde la familia hacia los pares y amigos, lo cual puede producir inestabilidad en las percepciones de apoyo. Así, Skinner y colaboradores (2000) observaron cambios en las percepciones de apoyo en adolescentes con diabetes, encontrando que el apoyo de pares era más importante que el apoyo de la familia en el caso de los adolescentes mayores.

Esta baja relación entre calidad de vida relacionada con la salud y apoyo social percibido en adolescentes con enfermedades crónicas es consistente con otros resultados obtenidos en nuestro medio respecto al escaso rol que tendría el apoyo social en el bienestar y la salud de adolescentes sanos. Así, Barra et al. (2005), utilizando una medida distinta de apoyo social a la utilizada en el presente estudio, encontraron que el apoyo social percibido tenía relaciones bajas tanto con los problemas de salud como con el estrés y el ánimo depresivo. $\mathrm{Y}$ en otro estudio con adolescentes sanos donde se utilizó la misma medida de apoyo social del presente estudio, Barra et al. (2006) encontraron que el apoyo social percibido simplemente no tenía relación ni con los problemas de salud informados por los adolescentes ni con los eventos estresantes, el estrés percibido ni el ánimo depresivo, todos los cuales presentaban una relación significativa con los problemas de salud.

Por lo tanto, según los resultados de este estudio y de los otros estudios mencionados, el apoyo social parece ser un factor más claramente relacionado con el bienestar y la salud en los adultos que en los adolescentes. La baja relación en la adolescencia entre apoyo social y factores como calidad de vida, salud o bienestar, podría sugerir que en esta etapa el apoyo social percibido sería un factor todavía no bien establecido y que, al igual que muchas otras dimensiones individuales, se sigue desarrollando al menos durante toda la etapa adolescente. Lo anterior tiene más sentido especialmente si se concibe el apoyo percibido como una disposición o una característica relativamente estable de personalidad (Dolbier \& Steinhardt, 2000; Gracia \& Herrero, 2004), más que como sólo el resultado de los niveles específicos de apoyo recibido o de las características del ambiente social.

En cuanto a las posibles diferencias de género, los resultados indican que las mujeres adolescentes informan menor nivel de calidad de vida que los hombres en la mayoría de las dimensiones, aunque la diferencia de género sólo era significativa en la dimensión de bienestar físico. Este resultado es consistente con lo encontrado por Arrington et al. (2006) en una muestra de adolescentes con fibrosis quística y por Petersen et al. (2006) en niños y adolescentes con diversas enfermedades crónicas. El que se observe una diferencia de 
género significativa en la dimensión de bienestar físico de los adolescentes es consistente también con otros estudios realizados en nuestro medio (Barra et al., 2005, 2006), donde se reporta que las mujeres adolescentes informan más quejas somáticas que los hombres. Ello puede explicarse porque ellas presentan también mayores niveles de estrés y ánimo depresivo, dos variables que tendrían una influencia significativa en la percepción y reporte de síntomas físicos, aspectos relacionados con en el bienestar físico.

En cuanto a la edad se encontró una relación negativa con la calidad de vida, lo que indica que a menor edad mayor calidad de vida. Aunque la evidencia al respecto es escasa y por tanto es difícil la comparación con otros estudios, los resultados del presente estudio serían consistentes con lo encontrado por Petersen y colaboradores (2006), quienes en una muestra de niños y adolescentes con tres enfermedades crónicas diferentes, observaron un efecto significativo de la edad en la dimensión emoción de la calidad de vida, siendo los participantes menores los que presentaban mayor puntaje. Este resultado podría ser explicado desde una perspectiva evolutiva, al relacionarlo con las tareas del desarrollo, la transición física y social que ocurre en el adolescente, la emergencia de grupos sociales y de pares, y la importancia que adquieren los cambios a nivel físico, los cuales pueden afectar la imagen corporal y la autoestima.

Los resultados obtenidos en el presente estudio subrayan la importancia de los factores cognitivos y conductuales en la calidad de vida, ya que muestran que algunos individuos pueden percibir una buena calidad de vida aun padeciendo una enfermedad crónica, con todo lo que ella implica desde el punto de vista físico. Por lo cual se puede plantear que la calidad de vida relacionada con la salud depende en un grado importante de algunos factores personales y del establecimiento de una determinada relación con el contexto social y el ambiente externo, en todo lo cual la autoeficacia puede desempeñar un rol fundamental.

Debido a que las enfermedades crónicas pueden ser progresivas y a que los avances en diagnóstico y tratamiento se traducen en una cada vez mayor sobreviva, la atención de la enfermedad debería incluir, además del tratamiento físico, intervenciones psicoeducativas con el objetivo de reforzar la autoeficacia de los pacientes y de esa manera incrementar su calidad de vida. Al respecto existen evidencias de que la implementación de programas de automanejo de la enfermedad crónica puede incrementar y reforzar la autoeficacia de los pacientes. Así, Lorig, Ritter y Jacquez (2005) encontraron que un programa de ese tipo de 6 semanas produjo mejorías en las conductas de salud, el estado de salud y la autoeficacia tanto a los 4 meses como al año, y tanto la autoeficacia basal como el aumento de autoeficacia a los 4 meses eran predictores significativos de las conductas de salud y el estado de salud al año después, por lo cual los autores concluyen que la autoeficacia sería un aspecto clave en el automanejo de la enfermedad crónica.
Sin embargo, también se debe considerar que la autoeficacia sólo explica una parte de las variaciones en la calidad de vida, por lo que futuras investigaciones deberían considerar nuevas variables personales, familiares y sociales que pueden tener relación con la calidad de vida y otros indicadores de salud.

Los resultados del presente estudio pueden aportar al desarrollo e implementación de estrategias de intervención orientadas a la promoción de aquellas características que favorecerían el logro de resultados de salud positivos y de una adecuada calidad de vida en las personas que deben vivir con enfermedades crónicas.

\section{Referencias}

Arnold, R., Ranchor, A., DeJongste, M., Koeter, G., Ten Hacken, N., Aalbers, R. et al. (2005). The relationship between self-efficacy and selfreported physical functioning in chronic obstructive pulmonary disease and chronic heart failure. Behavioral Medicine, 31 (3), 107-115.

Arrington-Sanders, R., Yi, M., Tsevat, J., Wilmott, R., Mrus, J. \& Britto, M. (2006). Gender differences in health-related quality of life of adolescents with cystic fibrosis. Health and Quality of Life Outcomes, 4, 1-5 (Publicado en línea y disponible en http://www.hqlo.com/content/4/1/5).

Barra, E. (2004). Apoyo social, estrés y salud. Psicología y Salud, 14 (2), 237-243.

Barra, E., Cancino, V., Lagos, G., Leal, P. \& San Martín, J. (2005). Factores psicosociales y problemas de salud reportados por adolescentes. Psicología y Salud, 15 (2), 231-239.

Barra, E., Cerna, R., Kramm, D. \& Véliz, V. (2006). Problemas de salud, estrés, afrontamiento, depresión y apoyo social en adolescentes. Terapia Psicológica, 24 (1), 55-61.

Bandura, A., Pastorelli, C., Barbaranelli, C. \& Caprara, G. (1999). Selfefficacy pathways to childhood depression. Journal of Personality and Social Psychology, 76 (2), 258-269.

Bishop, M. (2005). Quality of life and psychosocial adaptation to chronic illness and disability: Preliminary analysis of a conceptual and theoretical synthesis. Rehabilitation Counseling Bulletin, 48 (4), 219- 231.

Caprara, G., Steca, P., Gerbino, M., Paciello, M. \& Vecchio, G. (2006). Looking for adolescents' well-being: Self-efficacy beliefs as determinants of positive thinking and happiness. Epidemiologia e Psichiatria Sociale, 15 (1), 30-43

Dolbier, C. \& Steinhardt, M. (2002). The development and validation of the Sense of Support Scale. Behavioral Medicine, 25, 169-179

Frey, C. \& Röthlisberger, C. (1996). Social support in healthy adolescents. Journal of Youth and Adolescence, 25 (1), 17-31.

Gecková, A., Van Dijk, J., Stewart, R., Groothoff, J. \& Post, D. (2003). Influence of social support on health among gender and socio-economic groups of adolescents. European Journal of Public Health, 13 (1), 44-50.

Gielen, A., McDonnell, K., Wu, A., O'Campo, P. \& Faden, R. (2001). Quality of life among women living with HIV: The importance violence, social support, and self care behaviors. Social Science Medicine, 52 (2), 315-322

Gracia, E. \& Herrero, J. (2004). Personal and situational determinants of relationship-specific perceptions of social support. Social Behavior and Personality, 32, 459-476

Gracia, E., Herrero, J. \& Musitu, G. (2002). Evaluación de recursos y estresores psicosociales en la comunidad. Madrid: Editorial Síntesis.

Grau, C. (2005). Intervención temprana en niños con enfermedades crónicas. En Gómez, A., Viguer, P. \& Cantero, M. Intervención temprana (pp. 337-356). Madrid: Ediciones Pirámide.

Grey, M., Boland, E., Yu, C., Sullivan-Bolyai, S. \& Tamborlane, W. (1998). Personal and family factors associated with quality of life in adolescents with diabetes.Diabetes Care, 21 (6), 909-914. 
Han, K., Lee, P., Lee, S. \& Park, E. (2003). Factors influencing quality of life in people with chronic illness in Korea. Journal of Nursing Scholarship, 35 (2), 139-144.

Kohler, C., Fish, L. \& Greene, P. (2002). The relationship of perceived self-efficacy to quality of life in chronic obstructive pulmonary disease. Health Psychology, 21 (6), 610-614.

Kuijer, R. \& De Ridder, D. (2003). Discrepancy in illness-related goals and quality of life in chronically ill patients: The role of self-efficacy. Psychology and Health, 18, 313-330.

Livneh, H. \& Antonak, R. (2005). Psychosocial adaptation to chronic illness and disability: A primer for counselors. Journal of Counseling \& Development, 83 (1), 12-20.

Lorig K., Ritter P. \& Jacquez, A. (2005). Outcomes of border health Spanish/English chronic disease self-management programs. The Diabetes Educator, 3, 401-409.

Luszczynska, A., Gutiérrez, B. \& Schwarzer, R. (2005). General selfefficacy in various domains of human functioning: Evidence from five countries. International Journal of Psychology, 40 (2), 80-89.

Malkowska, A., Mazur, J. \& Woynarowska, B. (2004). Level of perceived social support and quality of life in children and adolescents aged 8-18 years. Medycyna Wieku Rozwojowego, 8 (3), 551-566.

Pastorelli, C., Caprara, G., Barbaranelli, C., Rola, J., Rozsa, S. \& Bandura, A. (2001). The structure of children's perceived self-efficacy: A cross-national study. European Journal of Psychological Assessment, 17 (2), 87-97.

Pendley, J., Kasmen, L., Miller, D., Donze, J., Swenson, C. \& Reeves, G. (2002). Peer and family support in children and adolescents with type 1 diabetes. Journal of Pediatric Psychology, 27 (5), 429-438.

Petersen, C., Schmidt, S. \& Bullinger, M. (2006). Coping with a chronic pediatric health condition and health-related quality of life. European Psychologist, 11(1), 50-56.

Ravens-Sieberer, U., Gosch, A., Rajmil, L., Erhart, M., Bruil, J., Duer, W. et al. (2005). KIDSCREEN-52 quality-of-life measure for children and adolescents. Expert Review of Pharmacoeconomics \& Outcomes Research, 5 (3), 353-364.

Rose, M., Fliege, H., Hildebrandt, M., Schirop, T. \& Klapp, B. (2002). The network of psychological variables in patients with diabetes and their importance for quality of life and metabolic control. Diabetes Care, 25 (1), 35-42.
Schwarzer, R. \& Scholz, U. (2000, Agosto). Cross-cultural assessment of coping resources: The General Perceived Self-efficacy Scale. Trabajo presentado en el Primer Congreso Asiático de Psicología de la Salud. Tokyo.

Skinner, T., John, M. \& Hampson, S. (2000). Social support and personal models of diabetes as predictors of self-care and well-being: A longitudinal study of adolescents with diabetes. Journal of Pediatric Psychology, 25 (4), 257-267

Suris, J., Michaud, P. \& Viner, R. (2004). The adolescent with a chronic condition. Part I: Developmental issues. Archives of Disease Childhood, 89 (10), 938-942.

Uchino, B. (2006). Social support and health: A review of physiological processes potentially underlying links to disease outcomes. Journal of Behavioral Medicine, 29(4), 377-87

Vázquez, I., Valderrábano, F., Jofré, R., Fort, J., López, J., Moreno, F. \& Sanz, D. (2003). Psychosocial factors and quality of life in young hemodialysis patients with low comorbidity. Journal of Nephrology, 16 (6), 886-894

Wahl, A., Rustoen, T., Hanestad, B., Gjengedal, E. \& Moum, T. (2005). Self-efficacy, pulmonary function, perceived health and global quality of life of cystic fibrosis patients. Social Indicators Research, 72 , 239-261.

Woodgate, R. (2006). The importance of being there: Perspectives of social support by adolescents with cancer. Journal of Pediatric Oncology Nursing, 23 (3), 122-134.

Wu, A., Tang, C. \& Kwok, T. (2004). Self-efficacy, health locus of control, and psychological distress in elderly Chinese women with chronic illnesses. Aging Mental Health, 8 (1), 21-28.

Yeo, M. \& Sawyer, S. (2005). Chronic illness and disability. British Medical Journal, 330, 721-723. 